\title{
Cardiovascular and neuropsychiatric risks of varenicline and bupropion in smokers with chronic obstructive pulmonary disease
}

\author{
Daniel Kotz, ${ }^{1,2,3,4}$ Wolfgang Viechtbauer, ${ }^{5}$ Colin R Simpson, ${ }^{3}$ \\ Onno C P van Schayck, ${ }^{2,3}$ Robert West, ${ }^{4}$ Aziz Sheikh ${ }^{2,3,6}$
}

- Additional material is published online only. To view please visit the journal online (http://dx.doi.org/10.1136/ thoraxjnl-2017-210067)

For numbered affiliations see end of article.

Correspondence to Professor Daniel Kotz, Addiction Research and Clinical Epidemiology Unit, Medical Faculty of the Heinrich-Heine, University Düsseldorf, Institute of General Practice, Düsseldorf 40225, Germany;

Daniel.Kotz@med.uni-

duesseldorf.de

Received 26 January 2017

Revised 17 March 2017

Accepted 4 April 2017

Published Online First

4 May 2017

\section{ABSTRACT}

Background Varenicline and bupropion are effective smoking cessation treatments, but there are concerns about their safety in smokers with COPD.

Objective To investigate whether varenicline and bupropion are associated with serious adverse cardiovascular and neuropsychiatric events in smokers with COPD.

Methods In a retrospective cohort study, we used data from 14350 patients with COPD included in the QResearch database, which holds data from 753 National Health Service general practices across England. We identified patients with COPD who received a prescription of nicotine replacement therapy (NRT; $\mathrm{N}=10$ 426; reference group), bupropion $(\mathrm{N}=350)$ or varenicline $(\mathrm{N}=3574)$ in the period between January 2007 and June 2012. Patients were followed up for 6 months to compare incident cardiovascular (ie, ischaemic heart disease, stroke, heart failure, peripheral vascular disease and cardiac arrhythmias) and neuropsychiatric (ie, depression and self-harm) events using Cox proportional hazards models, adjusted for potential confounders. Propensity score analysis was used as an additional approach to account for potential confounding by indication. We also modelled the effects of possible unmeasured confounders.

Results Neither bupropion nor varenicline showed an increased risk of adverse events compared with NRT. Varenicline was associated with a significantly reduced risk of heart failure $(\mathrm{HR}=0.56,95 \% \mathrm{Cl} 0.34$ to 0.92$)$ and depression ( $\mathrm{HR}=0.73,95 \% \mathrm{Cl} 0.61$ to 0.86$)$. Similar results were obtained from the propensity score analysis. Modelling of unmeasured confounding provided additional evidence that an increased risk of these adverse events was very unlikely.

Conclusion In smokers with COPD, varenicline and bupropion do not appear to be associated with an increased risk of cardiovascular events, depression or self-harm in comparison with NRT.

\section{INTRODUCTION}

Varenicline and bupropion are effective smoking cessation treatments, but have been suspected to be associated with serious adverse cardiovascular and neuropsychiatric events. The two drugs have proved to be effective in aiding long-term smoking cessation, both in the general smoking population ${ }^{12}$ and in the subgroup of smokers with COPD. ${ }^{3}$ However, postmarketing reports raised concerns

\section{Key messages}

What is the key question?

- Are varenicline or bupropion associated with an increased risk of serious cardiovascular events, depression or self-harm in smokers with COPD?

What is the bottom line?

- In smokers with COPD, varenicline and bupropion do not appear to be associated with an increased risk of documented cardiovascular events, depression or self-harm.

\section{Why read on?}

- This is the first study investigating the most important serious neuropsychiatric and cardiovascular adverse events in one study and with the same rigorous methodology in a large sample ( $\mathrm{N}=14350)$ of patients with COPD using real-life data.

about the risk of serious adverse cardiovascular and neuropsychiatric events, prompting the US Food and Drug Administration (FDA) and the European Medicines Agency to add warnings in the drugs' prescribing information. This included a boxed warning-FDA's most prominent warning-in the drug labels in 2009 about symptoms such as depressed mood, suicidal thoughts and behaviour and attempted suicide. ${ }^{4}$

Very recently, the FDA decided to remove the boxed warning for serious mental health effects from the varenicline and bupropion labels. ${ }^{5}$ This decision was based on a large randomised controlled trial that the FDA required the drug companies to conduct, which showed no significant increase in neuropsychiatric events in users of varenicline or bupropion compared with users of nicotine patch or placebo. ${ }^{6}$ The trial thereby confirmed evidence from meta-analyses of previous randomised controlled trials and from observational studies indicating that a causal relationship between the use of these drugs and serious adverse cardiovascular and neuropsychiatric events is unlikely. ${ }^{7-11}$ One of these studies was an observational study we conducted using analysis of a large, validated English primary care database. ${ }^{11}$ 
Although there is now good evidence about the safety of varenicline and bupropion in the general smoking population, it is important to assess specifically whether these drugs are associated with serious adverse events in diseased subgroups, particularly in smokers with COPD who are already, by virtue of their diagnosis, at increased risk of cardiovascular and neuropsychiatric events. ${ }^{12}$ Tobacco smoking is the most important risk factor for the development and progression of COPD, ${ }^{13}{ }^{14}$ and smoking cessation is the only treatment with proven effectiveness to reduce the accelerated decline in lung function. ${ }^{13}{ }^{14}$ It also affects other outcomes positively-for example, the response to treatment with bronchodilators and inhaled corticosteroids. ${ }^{14}$ International guidelines therefore recommend that smokers with COPD should be assisted during their attempt to quit with a combination of pharmacotherapy and behavioural support. ${ }^{13}{ }^{15}$ In UK and German primary care, varenicline is the preferred pharmacotherapy in smokers with COPD. ${ }^{16} 17$

In our previous study, ${ }^{11}$ we showed that varenicline and bupropion were not associated with an increased risk of serious adverse cardiovascular and neuropsychiatric events in the general smoking population. The aim of this study, as detailed in our a priori published study protocol, ${ }^{18}$ was to investigate the safety of the two drugs in the subgroup of smokers with COPD.

\section{METHODS}

We conducted a national, retrospective cohort study using the QResearch database (V.36, upload 31 July 2013), which holds anonymised health records of over 13 million patients from 753 National Health Service general practices from across England (http://www.qresearch.org). QResearch has been used for various studies of the incidence and risk of neuropsychiatric and cardiovascular events-in particular, our previous study on the safety of varenicline and bupropion in the general smoking population. ${ }^{11}$ We have now used this database to investigate the risk of the two drugs in the subgroup of smokers with COPD - a specific aim we described earlier in our study protocol. ${ }^{18}$ That protocol provides a detailed description of our analysis plan, and here we present below an overview of our methods. The only deviation from our published plan is that we could not perform an instrumental variable analysis because we were unable to identify a valid instrumental variable and instead, we undertook additional analyses (ie, modelling) to assess the impact of any potential unmeasured confounding. The use of this particular method was also prompted by concerns recently raised by the FDA in relation to evidence from previous observational studies on the safety of varenicline. ${ }^{19}$

\section{Inclusion and exclusion criteria}

We studied adult patients with recorded COPD who received prescriptions for varenicline, bupropion or nicotine replacement therapy (NRT) between 1 January 2007 and 30 June 2012. COPD was defined by appropriate Read codes (a clinical coding system used by general practitioners in the UK; see Kotz et $a l^{18}$ ). Patients aged $<35$ years and with no recording of spirometry or Medical Research Council dyspnoea score ${ }^{20}$ were excluded (14.0\% of all patients with recorded COPD). The date of first prescription of one of these drugs defined the individual's entry date to the cohort. Patients were excluded if they had used one of the drugs during 12 months before the start date of the study or if they had received a prescription of a combination of these drugs during the follow-up period.

\section{Exposure measures}

Patients were categorised into three exposure groups: (1) varenicline alone, (2) bupropion alone or (3) nicotine replacement therapy (NRT) alone, based on the drug they were first prescribed. We used NRT as a reference group to reduce the risk of confounding by indication ${ }^{18}$ and because it is presumed by regulators not to carry serious risks. In the UK, all three drugs are licensed only for use to aid smoking cessation. ${ }^{21}$ Start of follow-up began for each patient on the date of the first prescription and ended after 6 months' follow-up or when reaching the specific event of interest (see below). Patients who were lost to follow-up because they left the practice or died were censored on that date.

\section{Outcome measures}

We separately considered major incident neuropsychiatric and cardiovascular events that occurred during 6 months of follow-up for which a potential association with varenicline use has been suggested. ${ }^{1022} 23$ The cardiovascular events of interest were ischaemic heart disease, stroke, heart failure, peripheral vascular disease and cardiac arrhythmias. The neuropsychiatric outcomes of interest were depression and fatal or non-fatal selfharm. A follow-up period of 6 months covers the treatment duration of the drugs (typically 12 weeks) as well as an extended period after termination of treatment, in which many of the spontaneously reported adverse events occurred and where the excess in cardiovascular events was found in meta-analyses of clinical trials. As a secondary outcome, we assessed the occurrence of these events during the first 3 months of follow-up.

\section{Confounding factors}

The following variables, measured at or before the patient's entry date to the cohort, were included in the analyses as potential confounders: age, sex, socioeconomic status (measured using the Townsend Index ${ }^{24}$ ), Medical Research Council dyspnoea score, ${ }^{20}$ Strategic Health Authority of the general practice, relevant comorbidities from the Charlson Index ${ }^{25}$ (ie, diabetes, peptic ulcer disease, renal disease, rheumatological disease or cancer) and alcohol misuse. In addition, any recordings of the neuropsychiatric and cardiovascular events of interest that occurred before the patient's entry date to the cohort were also included.

\section{Statistical analyses}

We used Cox proportional hazards regression models to assess the association between exposure group and each of the above mentioned events, adjusted for all measured potential confounders (see above). All variables were entered as binary variables into the models except for the continuous variables age and socioeconomic status. We also used a propensity score analysis with trimming and matching to account for potential confounding by indication (the methodological details of this analysis have been reported in our study protocol ${ }^{18}$ ). In addition, we used an approach described by Lin $e t a l^{26}$ to model the effects of any potential unmeasured confounding. For this purpose, we adjusted the HRs and 95\% CIs in users of varenicline versus NRT for each of the events for a hypothetical, unmeasured, binary confounder with a HR of 3 and various combinations of prevalence among the two exposure groups.

All analyses were undertaken in R (V.3.0.2 or later). We provide the codes used in $\mathrm{R}$ as online supplementary material text E1. All statistical tests were two-sided with $\mathrm{p}<0.05$ indicating significance. 


\section{Ethical considerations}

This study involved the analysis of anonymised, routinely collected data. Our protocol was independently peer reviewed by the QResearch Scientific Board and satisfied the requirements of the Trent research ethics committee.

\section{RESULTS}

A total of 14350 patients with COPD were included in the analyses: 10426 users of NRT, 350 users of bupropion and 3574 users of varenicline (figure 1). This subgroup of smokers with COPD from the database were older, more deprived and showed higher prevalence rates of comorbid diseases, including the cardiovascular and neuropsychiatric diseases of interest, than the subgroup of smokers without COPD (table 1).

Patients with COPD who used NRT were older, had more severe dyspnoea and showed higher prevalence rates of comorbid diseases than users of bupropion and varenicline (table 2). The highest incidence rates of events were found for depression and ischaemic heart disease (table 3, see online supplementary figures E1-7).

\section{Cox proportional hazards regression analyses}

Neither bupropion nor varenicline showed an increased risk of any cardiovascular or neuropsychiatric event compared with NRT (table 3). The estimated HR were $<1$ in users of bupropion and varenicline relative to NRT users-indicating no increased risk-for stroke, heart failure, peripheral vascular disease, arrhythmia, depression or self-harm. Only for ischaemic heart disease, the HR was $>1$ in users of bupropion and varenicline, but this difference was not statistically significant (for varenicline, the HR was only minimally higher than 1 and the $\mathrm{CI}$ was large: $H R=1.02,95 \%$ CI 0.83 to 1.24 ). Rather, varenicline was associated with a significantly reduced risk of heart failure $(\mathrm{HR}=0.56,95 \% \mathrm{CI} 0.34$ to 0.92$)$ and depression $(\mathrm{HR}=0.73$, $95 \%$ CI 0.61 to 0.86 ).
Figure 1 Flow chart. NRT, nicotine replacement therapy; PS, propensity score.

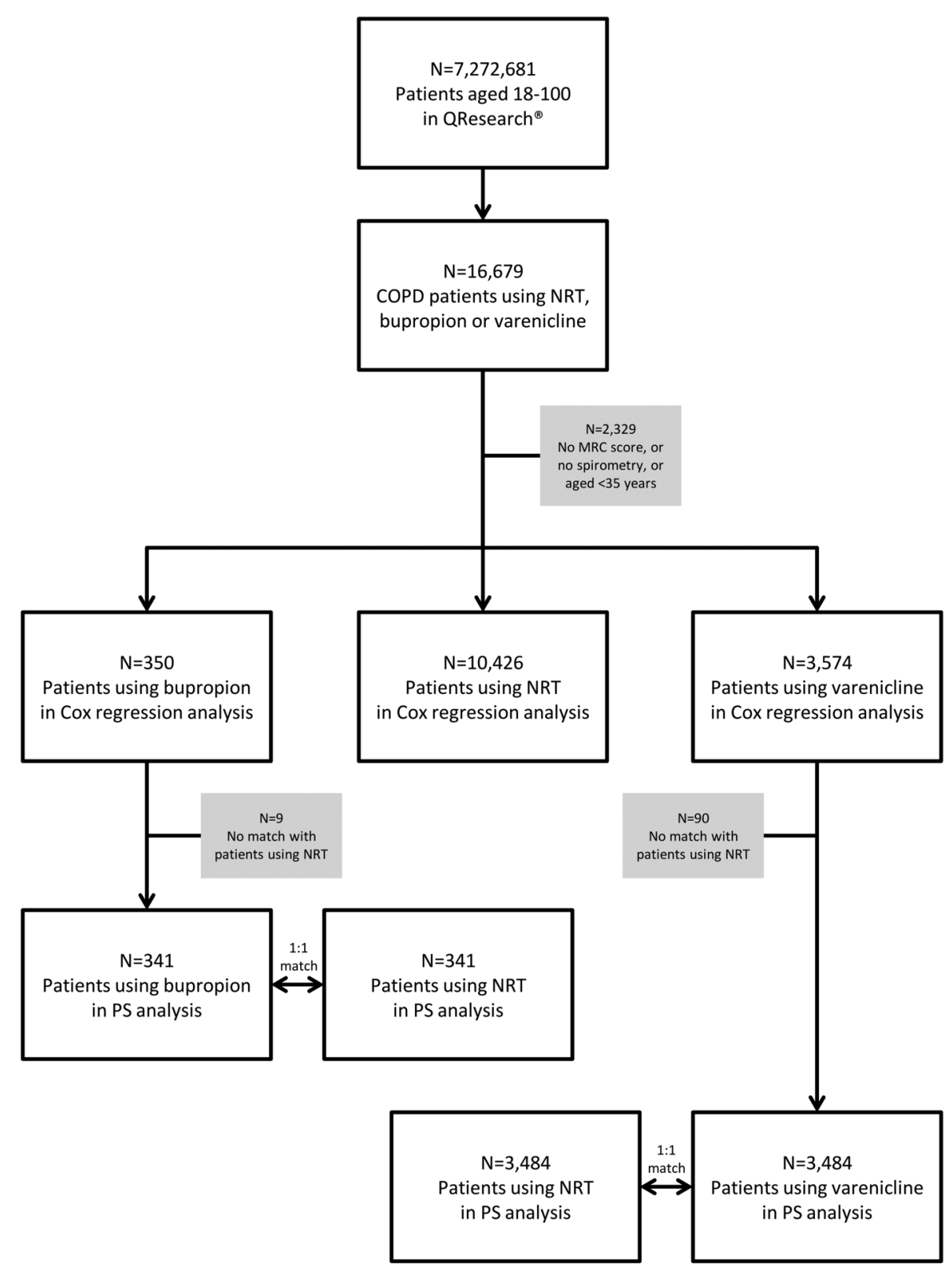


Table 1 Characteristics of the subgroups of patients with (current study population) and without COPD at entry date to the cohort

\begin{tabular}{lcc}
\hline Characteristics & \multicolumn{1}{c}{ COPD } & No COPD \\
(N=14 350) & (N=150 416) \\
\hline Age, mean (SD) & $54.70(9.59)$ & $38.15(12.31)$ \\
Female sex & $7377(51.41)$ & $75826(50.41)$ \\
Socioeconomic status*, mean (SD) & $3.25(1.35)$ & $3.09(1.35)$ \\
Diabetes & $1508(10.51)$ & $8647(5.75)$ \\
Peptic ulcer disease & $1078(7.51)$ & $3635(2.42)$ \\
Renal disease & $1227(8.55)$ & $5018(3.34)$ \\
Rheumatological disease & $888(6.19)$ & $3278(2.18)$ \\
Cancer & $1249(8.70)$ & $4670(3.10)$ \\
Alcohol misuse & $1431(9.97)$ & $10535(7.00)$ \\
Prior ischaemic heart disease & $2018(14.06)$ & $6046(4.02)$ \\
Prior stroke & $1073(7.48)$ & $3353(2.23)$ \\
Prior heart failure & $388(2.70)$ & $671(0.45)$ \\
Prior peripheral vascular disease & $531(3.70)$ & $1277(0.85)$ \\
Prior arrhythmia & $742(5.17)$ & $2337(1.55)$ \\
Prior depression & $5545(38.65)$ & $53167(35.35)$ \\
Prior self-harm & $1563(10.89)$ & $15711(10.45)$ \\
\hline
\end{tabular}

Data are presented as $\mathrm{N}$ (percentage within drug group) unless stated otherwise.

*Townsend Index: 1 (lowest) to 5 (highest level of deprivation). ${ }^{24}$

Table 2 Characteristics of patients with COPD at entry date to the cohort, stratified by medication group

\begin{tabular}{|c|c|c|c|}
\hline Characteristics & $\begin{array}{l}\text { NRT } \\
(N=10426)\end{array}$ & $\begin{array}{l}\text { Bupropion } \\
(\mathrm{N}=350)\end{array}$ & $\begin{array}{l}\text { Varenicline } \\
(\mathrm{N}=3574)\end{array}$ \\
\hline Age, mean (SD) & $55.53(9.72)$ & $52.42(9.31)$ & $52.48(8.82)$ \\
\hline Female sex & $5390(51.70)$ & $165(47.14)$ & $1822(50.98)$ \\
\hline $\mathrm{MRC}^{*}$ score, mean (SD) & $2.37(1.02)$ & $2.22(0.96)$ & $2.23(0.98)$ \\
\hline $\begin{array}{l}\text { Socioeconomic statust, } \\
\text { mean (SD) }\end{array}$ & $3.28(1.35)$ & $3.14(1.31)$ & $3.20(1.37)$ \\
\hline Diabetes & $1163(11.15)$ & $20(5.71)$ & $325(9.09)$ \\
\hline Peptic ulcer disease & $819(7.86)$ & $23(6.57)$ & $236(6.60)$ \\
\hline Renal disease & 979 (9.39) & $14(4.00)$ & $234(6.55)$ \\
\hline Rheumatological disease & $679(6.51)$ & $13(3.71)$ & $196(5.48)$ \\
\hline Cancer & $943(9.04)$ & $29(8.29)$ & $277(7.75)$ \\
\hline Alcohol misuse & $1112(10.67)$ & $22(6.29)$ & $297(8.31)$ \\
\hline Prior ischaemic heart disease & $1556(14.92)$ & $32(9.14)$ & $430(12.03)$ \\
\hline Prior stroke & $829(7.95)$ & $22(6.29)$ & $222(6.21)$ \\
\hline Prior heart failure & $315(3.02)$ & $5(1.43)$ & $68(1.90)$ \\
\hline $\begin{array}{l}\text { Prior peripheral vascular } \\
\text { disease }\end{array}$ & $416(3.99)$ & $6(1.71)$ & 109 (3.05) \\
\hline Prior arrhythmia & $603(5.78)$ & $13(3.71)$ & $126(3.53)$ \\
\hline Prior depression & 4149 (39.79) & $132(37.71)$ & $1264(35.37)$ \\
\hline Prior self-harm & $1174(11.26)$ & $32(9.14)$ & 357 (9.99) \\
\hline
\end{tabular}

Chi-squared tests indicated that hazards were not proportional for the outcome depression $(p=0.041)$, but a more finegrained analysis allowing for varying HRs indicated that the HR always fell below 1.00 across the entire follow-up period. Furthermore, we found that the risk of heart failure in users of varenicline compared with NRT differed statistically between female and male subjects $(p=0.017)$, but the HR was again always $>1.00$.

\section{Propensity score analyses}

After trimming and matching patients by propensity score, the sample size was 682 for the comparison of bupropion versus NRT and 6968 for the comparison of varenicline versus NRT. A comparison of patient characteristics showed that the drug groups were generally well matched in both comparisons (see online supplementary table E1). Neither bupropion nor varenicline showed an increased risk of any neuropsychiatric or cardiovascular event compared with NRT (table 4). For ischaemic heart disease, the HR was higher than 1 in users of bupropion and lower in users of varenicline, but in both cases the difference was small and not statistically significant $(\mathrm{HR}=1.23,95 \%$ CI 0.49 to 3.12 for bupropion vs NRT and $\mathrm{HR}=0.97,95 \% \mathrm{CI}$ 0.75 to 1.24 for varenicline vs NRT). Also in this propensity score analysis, varenicline was associated with a significantly reduced risk of depression $(\mathrm{HR}=0.68,95 \% \mathrm{CI} 0.55$ to 0.83$)$.

\section{Modelling of unmeasured confounding}

The modelling showed that an increased risk of any of the neuropsychiatric and cardiovascular events in users of varenicline was very unlikely (see online supplementary tables E2-8). For example, an unmeasured confounder with a HR of 3 for self-harm would have reversed the observed reduced HR in users of varenicline versus NRT $(\mathrm{HR}=0.78)$ into an increased HR $(>1.00)$ only if the prevalence of this confounder had been very different among the two groups of medication users (eg, only $0 \%$ among users of varenicline and simultaneously at least $90 \%$ among users of NRT; table 5).

\section{Secondary analyses}

The results from the Cox proportional hazards regression analyses, propensity score analyses and the modelling of unmeasured confounding with the occurrence of the cardiovascular and neuropsychiatric events during 3 months of follow-up yielded very similar results (see online supplementary tables E9-17).

\section{DISCUSSION}

We found no evidence for any increased risk of cardiovascular and neuropsychiatric adverse events in smokers with COPD using varenicline or bupropion to aid their attempt to quit in comparison with users of NRT. On the contrary, some events were associated with a reduced risk (ie, heart failure and depression). Modelling the effects of any potential unmeasured confounders found that these would only lead to an increased risk associated with varenicline use under unlikely assumptions.

We are not aware of previous studies specifically designed to assess the risks of varenicline or bupropion in patients with COPD. The efficacy trial by Tashkin et $a l^{27}$ found higher rates of psychiatric adverse events (in particular, sleep and mood disorders), but no difference in serious adverse events between active varenicline and placebo. However, this trial included only 504 smokers and was not statistically powered to detect rare events.

More evidence on the risks of varenicline is available from studies conducted in the general smoking population. For cardiovascular events, one meta-analysis reported an increased risk of cardiovascular events in users of varenicline, ${ }^{23}$ whereas later meta-analyses ${ }^{7-9}$ and large-scale observational studies found no such association. ${ }^{10} 11$ With regard to neuropsychiatric events, a recent trial in 8144 smokers with and without psychiatric disorders found no significant increase in neuropsychiatric adverse events attributable to varenicline relative to nicotine patch or placebo. $^{6}$ Previous observational studies also found no 
Table 3 Incidence rates of events and HRs $(95 \% \mathrm{Cl})$ of medication groups for all events during 6 months' follow-up

\begin{tabular}{|c|c|c|c|c|c|}
\hline \multirow[b]{2}{*}{ Event } & \multirow[b]{2}{*}{ Patient-years } & \multirow[b]{2}{*}{ Number of events } & \multirow{2}{*}{$\begin{array}{l}\text { Incidence of event } \\
\text { per } 1000 \text { patient-years }\end{array}$} & \multicolumn{2}{|l|}{$\mathrm{HR}(95 \% \mathrm{CI})$} \\
\hline & & & & Crude & Adjusted* \\
\hline \multicolumn{6}{|c|}{ Ischaemic heart disease } \\
\hline NRT & 5061 & 417 & 82.4 & 1 & 1 \\
\hline Bupropion & 172 & 11 & 64.0 & $0.78(0.43$ to 1.42$)$ & $1.18(0.64$ to 2.15$)$ \\
\hline Varenicline & 1756 & 128 & 72.9 & $0.89(0.73$ to 1.08$)$ & $1.02(0.83$ to 1.24$)$ \\
\hline \multicolumn{6}{|l|}{ Stroke } \\
\hline NRT & 5140 & 155 & 30.2 & 1 & 1 \\
\hline Bupropion & 175 & 3 & 17.1 & $0.57(0.18$ to 1.79$)$ & $0.62(0.20$ to 1.96$)$ \\
\hline Varenicline & 1780 & 34 & 19.1 & $0.63(0.44$ to 0.92$)$ & $0.76(0.52$ to 1.11$)$ \\
\hline \multicolumn{6}{|l|}{ Heart failure } \\
\hline NRT & 5148 & 118 & 22.9 & 1 & 1 \\
\hline Bupropion & 175 & 1 & 5.7 & $0.25(0.03$ to 1.79$)$ & $0.40(0.06$ to 2.89$)$ \\
\hline Varenicline & 1783 & 18 & 10.1 & $0.44(0.27$ to 0.72$)$ & $0.56(0.34$ to 0.92$)$ \\
\hline \multicolumn{6}{|c|}{ Peripheral vascular disease } \\
\hline NRT & 5156 & 93 & 18.0 & 1 & 1 \\
\hline Bupropion & 175 & 1 & 5.7 & $0.32(0.04$ to 2.28$)$ & $0.49(0.07$ to 3.56$)$ \\
\hline Varenicline & 1784 & 17 & 9.5 & $0.53(0.32$ to 0.89$)$ & $0.62(0.37$ to 1.05$)$ \\
\hline \multicolumn{6}{|l|}{ Arrhythmia } \\
\hline NRT & 5134 & 174 & 33.9 & 1 & 1 \\
\hline Bupropion & 174 & 4 & 23.0 & $0.68(0.25$ to 1.83$)$ & 0.92 (0.34 to 2.50$)$ \\
\hline Varenicline & 1777 & 38 & 21.4 & $0.63(0.44$ to 0.90$)$ & $0.84(0.59$ to 1.20$)$ \\
\hline \multicolumn{6}{|l|}{ Depression } \\
\hline NRT & 4989 & 686 & 137.5 & 1 & 1 \\
\hline Bupropion & 171 & 17 & 99.4 & $0.72(0.45$ to 1.17$)$ & $0.73(0.45$ to 1.18$)$ \\
\hline Varenicline & 1745 & 167 & 95.7 & 0.70 (0.59 to 0.83$)$ & 0.73 (0.61 to 0.86$)$ \\
\hline \multicolumn{6}{|l|}{ Self-harm } \\
\hline NRT & 5174 & 36 & 7.0 & 1 & 1 \\
\hline Bupropion & 175 & 1 & 5.7 & $0.82(0.12$ to 6.00$)$ & 0.90 (0.12 to 6.58$)$ \\
\hline Varenicline & 1786 & 9 & 5.0 & $0.72(0.35$ to 1.51$)$ & $0.78(0.37$ to 1.63$)$ \\
\hline
\end{tabular}

Table 4 HRs $(95 \% \mathrm{Cl})$ of events during 6 months' follow-up in the propensity score matched samples

\begin{tabular}{lll}
\hline & HR (95\% Cl) & \\
\cline { 2 - 3 } Event & $\begin{array}{l}\text { Bupropion vs NRT } \\
\text { (N=682) }\end{array}$ & $\begin{array}{l}\text { Varenicline vs NRT } \\
\text { (N=6968) }\end{array}$ \\
\hline Ischaemic heart disease & $1.23(0.49$ to 3.12$)$ & $0.97(0.75$ to 1.24$)$ \\
Stroke & $0.49(0.12$ to 1.95$)$ & $1.06(0.65$ to 1.75$)$ \\
Heart failure & $0.99(0.06$ to 15.89$)$ & $0.77(0.41$ to 1.45$)$ \\
Peripheral vascular disease & $0.99(0.06$ to 15.80$)$ & $0.57(0.31$ to 1.05$)$ \\
Arrhythmia & $3.96(0.44$ to 35.41$)$ & $1.12(0.70$ to 1.81$)$ \\
Depression & $0.75(0.40$ to 1.41$)$ & $0.68(0.55$ to 0.83$)$ \\
Self-harm & $0.49(0.04$ to 5.43$)$ & $0.60(0.26$ to 1.37$)$ \\
\hline
\end{tabular}

NRT, nicotine replacement therapy.

association between varenicline use and neuropsychiatric risks. ${ }^{11} 21222829$

Our study has several strengths and limitations. A study using observational data to compare the risks of different groups of medication users is prone to confounding by indication. Our data indeed show differences in patient characteristics between users of varenicline, bupropion and NRT. At baseline, the reference group of NRT users had higher prevalence rates of risk factors for the adverse events under study (eg, NRT users were older, had more severe COPD and higher prevalence of comorbidities). We accounted for such differences by adjusting our regression models with measured confounders and by reanalysing the data in a propensity score analysis with trimming and matching. Uniquely, we also modelled what would need to be the distribution and influence of unmeasured confounders to overturn the key conclusions. We applied this approach originally described by Lin et $a l,{ }^{26}$ also in our previous analyses on the risks of varenicline in the general smoking population and described the implications in more detail. ${ }^{11}$ In summary, we conclude that our findings are unlikely to be confounded to an extent that would have obscured an increased risk of varenicline.

Another point to discuss is the use of routinely collected data in this study. Our definition of COPD relied on diagnostic codes entered by general practitioners into the patients' electronic health records. We combined codes for COPD diagnosis with codes for the measurement of spirometry and dyspnoea and excluded patients under the age of 35 to increase the validity of our definition. However, we did not have individual patient data on lung function at the time of inclusion into our cohort. Hence, misclassification might have occurred and a more finegrained analysis according to different stages of COPD severity 


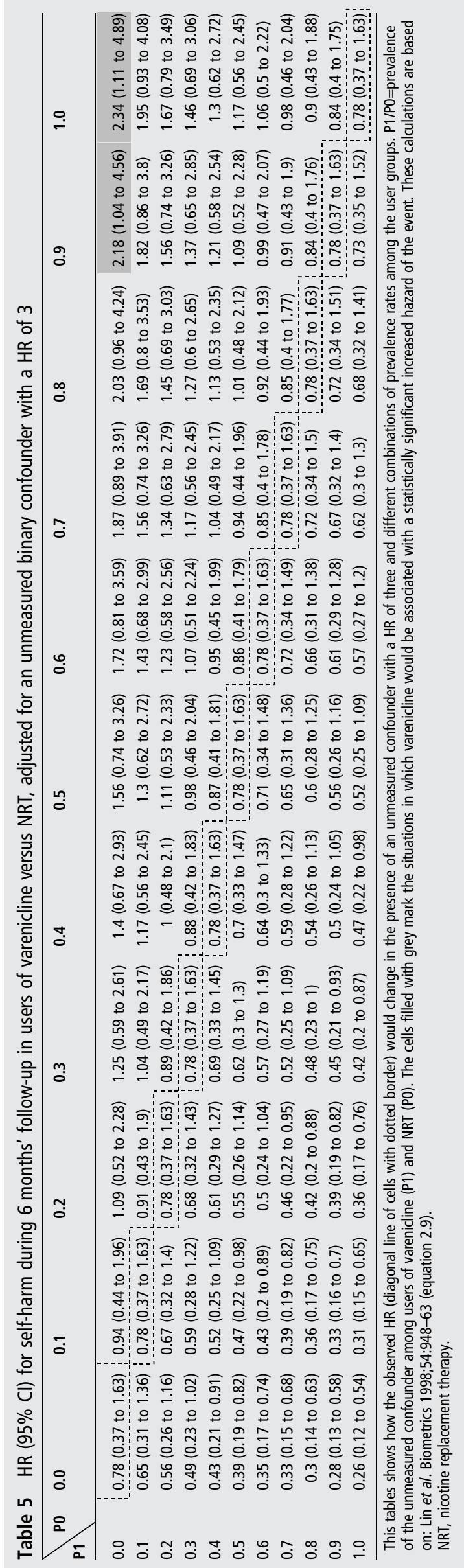

was not possible. Furthermore, this routine dataset did not include variables of potential interest, such as medication adherence, previous and current levels of tobacco exposure and smoking cessation outcomes during follow-up. We were also not able to assess what the FDA has described as 'nuanced' neuropsychiatric symptoms such as mood disorders that involve aggression. ${ }^{30}$ Nevertheless, the neuropsychiatric events in our study are among the most important ones and are included in the boxed warning. Finally, the sample size for the statistical analyses with bupropion was rather low as only 350 patients with COPD had used this medication, which resulted in large CIs around the HRs for some adverse events. Therefore, for those adverse events, estimated values from the statistical models need to be cautiously interpreted.

A major strength of this work is that we conducted the first, large-scale study on this topic in this patient population, with a sample size that included 3574 patients with COPD using varenicline (and 14350 patients with COPD in total). Second, we investigated the most important neuropsychiatric and cardiovascular adverse events at the same time and with the same methodology. Third, our study has high external validity due to the use of real-life patient data collected from a large number of different general practitioner practices across England (a country with a national healthcare system in which all members of the community, regardless of socioeconomic status, have free and ready access to smoking cessation treatment). Finally, we planned our study methodology and described it in great detail before the analysis and interpretation of data in a peer-reviewed protocol. ${ }^{18}$

We conclude that, in smokers with COPD, varenicline and bupropion are unlikely to be associated with increased risk of serious adverse neuropsychiatric or cardiovascular events compared with NRT.

\section{Author affiliations \\ ${ }^{1}$ Addiction Research and Clinical Epidemiology Unit, Medical Faculty of the Heinrich- Heine, Institute of General Practice, University Düsseldorf, Düsseldorf, Germany ${ }^{2}$ Department of Family Medicine, CAPHRI School for Public Health and Primary Care, Maastricht University Medical Centre, Maastricht, The Netherlands \\ ${ }^{3}$ Allergy \& Respiratory Research Group, Centre for Medical Informatics, Usher Institute of Population Health Sciences and Informatics, The University of Edinburgh, Edinburgh, UK \\ ${ }^{4}$ Cancer Research UK Health Behaviour Research Centre, University College London, London, UK \\ ${ }^{5}$ MHeNS School for Mental Health and Neuroscience, Maastricht University, Maastricht, The Netherlands \\ ${ }^{6}$ Division of General Internal Medicine and Primary Care, Brigham and Women's Hospital/Harvard Medical School, Boston, Massachusetts, USA}

Acknowledgements We acknowledge the contribution of EMIS practices who contribute to the QResearch, the University of Nottingham and EMIS for establishing, developing and supporting the database and supplying the data for this research project. We specifically thank Professor Julia Hippisley-Cox for her advice and support in conducting this study.

Contributors DK had the original idea for this study, drafted its funding application and this article. He had full access to all the data in the study and takes responsibility for the integrity of the data and the accuracy of the data analysis. WV and DK wrote the statistical script and analysed the data. All authors contributed to the conception and design of the study, revised the article and gave final approval of the version to be published.

Funding QInnovation Award (provided by the software provider EMIS and the University of Nottingham) with additional support from the Ministry for Innovation, Science and Research of the German Federal State of North Rhine-Westphalia ("NRW-Rückkehrprogramm)", Cancer Research UK, the Medical Research Council and The Commonwealth Fund. The funder provided access to the QResearch database, which included collection and management of data. The funder was not involved with the design and conduct of the study; analysis and interpretation of the data; preparation, review or approval of the manuscript; and decision to submit the manuscript for publication. 
Competing interests DK received an unrestricted grant from Pfizer for an investigator-initiated trial on the effectiveness of practice nurse counselling and varenicline for smoking cessation in primary care (Dutch Trial Register NTR3067). RW received grants, personal fees and non-financial support from Pfizer, GSK and $J \& J$ and personal fees from Novartis. OCPvS received an unrestricted research grant from Pfizer. None of this funding was directly related to this study. Furthermore, the manufacturers of varenicline and bupropion were not involved during any stage of this project. DK was supported by a research grant ("NRW-Rückkehrprogramm)" from the Ministry for Innovation, Science and Research of the German Federal State of North Rhine-Westphalia. AS was supported by the Farr Institute, which is supported by a consortia of funders led by the Medical Research Council and The Commonwealth Fund, a private independent foundation based in New York City; the views presented here are those of the author and not necessarily those of The Commonwealth Fund, its directors, officers or staff.

Ethics approval Our protocol was independently peer reviewed by the QResearch Scientific Board and satisfied the requirements of the Trent research ethics committee.

Provenance and peer review Not commissioned; externally peer reviewed.

\section{REFERENCES}

1 Cahill K, Stead LF, Lancaster T. Nicotine receptor partial agonists for smoking cessation. Cochrane Database Syst Rev 2012;(4):CD006103.

2 Hughes JR, Stead LF, Hartmann-Boyce J, et al. Antidepressants for smoking cessation. Cochrane Database Syst Rev 2014:(1):CD000031.

3 van Eerd EA, van der Meer RM, van Schayck OC, et al. Smoking cessation for people with chronic obstructive pulmonary disease. Cochrane Database Syst Rev 2016;(8):CD010744.

4 US Food and Drug Administration, FDA alert [July 1, 2009]: Information for Healthcare Professionals: Varenicline (marketed as Chantix) and Bupropion (marketed as Zyban, Wellbutrin and generics). http://www.fda.gov/Drugs/ DrugSafety/PostmarketDrugSafetylnformationforPatientsandProviders/ DrugSafetylnformationforHeathcareProfessionals/ucm 169986.htm (accessed 3 Jan 2017).

5 US Food and Drug Administration. Drug Safety Communication [December 16, 2016]: FDA revises description of mental health side effects of the stop-smoking medicines Chantix (varenicline) and Zyban (bupropion) to reflect clinical trial findings. http://www.fda.gov/Drugs/DrugSafety/ucm532221.htm (accessed 3 Jan 2017)

6 Anthenelli RM, Benowitz NL, West R, et al. Neuropsychiatric safety and efficacy of varenicline, bupropion and nicotine patch in smokers with and without psychiatric disorders (EAGLES): a double-blind, randomised, placebo-controlled clinical trial. Lancet 2016;387:2507-20.

7 Prochaska JJ, Hilton JF. Risk of cardiovascular serious adverse events associated with varenicline use for tobacco cessation: systematic review and meta-analysis. BMJ 2012;344:e2856.

8 Cahill K, Stevens S, Lancaster T. Pharmacological treatments for smoking cessation. JAMA 2014;311:193-4.

9 Sterling $\mathrm{LH}$, Windle $\mathrm{SB}$, Filion $\mathrm{KB}$, et al. Varenicline and adverse cardiovascular events: a systematic review and meta-analysis of randomized controlled trials. J Am Heart Assoc 2016;5:pii: e002849.

10 Svanström $H$, Pasternak $B$, Hviid A. Use of varenicline for smoking cessation and risk of serious cardiovascular events: nationwide cohort study. BMJ 2012;345: e7176.

11 Kotz D, Viechtbauer W, Simpson C, et al. Cardiovascular and neuropsychiatric risks of varenicline: a retrospective cohort study. Lancet Respir Med 2015;3:761-8.

12 Global Initiative for Chronic Obstructive Lung Disease. Global strategy for the diagnosis, management and prevention of chronic pulmonary disease (2017 report).
Global Initiative for Chronic Obstructive Lung Disease, Inc, 2017. http://goldcopd. org (accessed 4 Jan 2017)

13 Global Initiative for Chronic Obstructive Lung Disease. Global strategy for the diagnosis, management and prevention of chronic pulmonary disease. Updated 2015. NHLBI/WHO, 2015. http://www.goldcopd.com (accessed 20 Mar 2015).

14 Jiménez-Ruiz CA, Andreas $\mathrm{S}$, Lewis KE, et al. Statement on smoking cessation in COPD and other pulmonary diseases and in smokers with comorbidities who find it difficult to quit. Eur Respir J 2015;46:61-79.

15 Tønnesen P, Carrozzi L, Fagerström KO, et al. Smoking cessation in patients with respiratory diseases: a high priority, integral component of therapy. Eur Respir J 2007;29:390-417.

16 Huang $Y$, Lewis $S$, Britton J. Use of varenicline for smoking cessation treatment in UK primary care: an association rule mining analysis. BMC Public Health 2014;14:1-5.

17 Andreas S, Chenot JF, Diebold R, et al. Effectiveness of varenicline as an aid to smoking cessation in primary care: an observational study. Eur Addict Res 2013;19:47-54

18 Kotz D, Simpson C, Viechtbauer W, et al. Cardiovascular and neuropsychiatric safety of varenicline and bupropion compared with nicotine replacement therapy for smoking cessation: study protocol of a retrospective cohort study using the QResearch general practice database. BMJ Open 2014;4:e005281.

19 US Food and Drug Administration, FDA Briefing Document. Joint Meeting of the Psychopharmacologic Drugs Advisory Committee and Drug Safety and Risk Management Advisory Committee. October 16, 2014. Chantix and Serious Neuropsychiatric Adverse Events. http://www.fda.gov/downloads/Advisory Committees/CommitteesMeetingMaterials/Drugs/PsychopharmacologicDrugsAdvisory Committee/UCM418705.pdf) (accessed 20 May 2015).

20 Bestall JC, Paul EA, Garrod R, et al. Usefulness of the Medical Research Counci (MRC) dyspnoea scale as a measure of disability in patients with chronic obstructive pulmonary disease. Thorax 1999;54:581-6.

21 Thomas KH, Martin RM, Davies NM, et al. Smoking cessation treatment and risk of depression, suicide and self harm in the Clinical Practice Research Datalink: prospective cohort study. BMJ 2013;347:f5704

22 Gunnell D, Irvine D, Wise L, et al. Varenicline and suicidal behaviour: a cohort study based on data from the General Practice Research Database. BMJ 2009;339: b3805.

23 Singh S, Loke YK, Spangler JG, et al. Risk of serious adverse cardiovascular events associated with varenicline: a systematic review and meta-analysis. Can Med Assoc J 2011;183:1359-66.

24 Townsend P, Phillimore P, Beattie A. Health and deprivation: inequality and the North. London: Croom Helm, 1988.

25 Khan NF, Perera R, Harper S, et al. Adaptation and validation of the Charlson Index for Read/OXMIS coded databases. BMC Fam Pract 2010;11:1.

26 Lin DY, Psaty BM, Kronmal RA. Assessing the sensitivity of regression results to unmeasured confounders in observational studies. Biometrics 1998;54:948-63.

27 Tashkin DP, Rennard S, Hays JT, et al. effects of varenicline on smoking cessation in patients with mild to moderate COPD. Chest 2011;139:591-9.

28 Meyer TE, Taylor LG, Xie $\mathrm{S}$, et al. Neuropsychiatric events in varenicline and nicotine replacement patch users in the Military Health System. Addiction 2013;108:203-10.

29 Cunningham FE, Hur K, Dong D, et al. A comparison of neuropsychiatric adverse events during early treatment with varenicline or a nicotine patch. Addiction 2016;111:1283-92.

30 US Food and Drug Administration. Transcript for the 16 October 2014 Joint Meeting of the Psychopharmacologic Drugs Advisory Committee (PDAC) and the Drug Safety and Risk Management Advisory Committee (DSaRM). http://www.fda.gov/AdvisoryCommittees/CommitteesMeetingMaterials/Drugs/ PsychopharmacologicDrugsAdvisoryCommittee/ucm394880.htm (accessed 9 Jul 2015). 